\title{
A reincidência de tentativa de suicídio associada a severidade da sintomatologia
}

\section{depressiva}

\author{
The recidivism of suicide attempt associated with the severity of depressive symptoms \\ La recurrencia de un intento de suicidio asociado con la gravedad de los síntomas depresivos
}

Recebido: 24/11/2021 | Revisado: 02/12/2021 | Aceito: 02/12/2021 | Publicado: 12/12/2021

\author{
Daniel Augusto da Silva \\ ORCID: https://orcid.org/ 0000-0002-2716-6700 \\ Universidade Federal de São Paulo, Brasil \\ E-mail: daniel.augusto@unifesp.br \\ João Fernando Marcolan \\ ORCID: https://orcid.org/ 0000-0001-8881-7311 \\ Universidade Federal de São Paulo, Brasil \\ E-mail: jfmarcolan@uol.com.br
}

\begin{abstract}
Resumo
Pesquisa com o objetivo de analisar a associação entre a recorrência da tentativa de suicídio e a gravidade dos sintomas depressivos. Trata-se de uma pesquisa exploratória, descritiva, quantitativa, com coleta de dados realizada entre dezembro de 2017 e novembro de 2019, em uma Unidade de Pronto Atendimento de Assis/SP. Participaram 95 pessoas que compareceram por tentativa de suicídio, por meio de entrevista com questionário semiestruturado e aplicação do Inventário de Depressão de Beck. Para a análise dos dados, utilizou-se a estatística descritiva e inferencial, utilizando-se o teste exato de Fisher e o coeficiente de contingência V de Cramer. O nível de significância adotado foi $\mathrm{p}<0,05$. O projeto de pesquisa foi aprovado pelo Comitê de Ética em Pesquisa. Entre os 95 (100,0\%) participantes, $66(69,5 \%)$ tiveram recorrência da tentativa de suicídio e 36 (37,9\%) tinham diagnóstico prévio de depressão. Com a análise da associação entre a recorrência da tentativa de suicídio e os níveis de depressão, permitese afirmar que são variáveis dependentes tanto para pessoas sem depressão prévia $(\mathrm{p}=0,022)$ quanto para pessoas com depressão prévia $(\mathrm{p}=0,030)$. A recorrência para tentativa de suicídio foi prevalente para pessoas com níveis mais graves de sintomas depressivos.
\end{abstract}

Palavras-chave: Tentativa de suicídio; Reincidência; Depressão; Fatores de risco.

\begin{abstract}
Research aiming to analyze the association between recurrence of suicide attempt and the severity of depressive symptoms. This is an exploratory, descriptive, quantitative research, with data collection carried out between December 2017 and November 2019, in an Emergency Care Unit in Assis / SP. Participated 95 people who were in attendance due to suicide attempt, through interview with semi-structured questionnaire and application of the Beck Depression Inventory. For data analysis, descriptive and inferential statistics were used, using Fisher's exact test and Cramer's V contingency coefficient. The level of significance was set at $\mathrm{p}<0.05$. The research project was approved by the Research Ethics Committee. Among the 95 (100.0\%) participants, $66(69.5 \%)$ had a recurrence of suicide attempt, and $36(37.9 \%)$ had a previous diagnosis for depression. With the analysis of the association between the recurrence of suicide attempt and the levels of depression, it is allowed to state that they are dependent variables both for people without previous depression $(\mathrm{p}=0.022)$ and for people with previous depression $(\mathrm{p}=0.030)$. Recurrence for attempted suicide was prevalent for people with more severe levels of depressive symptoms.
\end{abstract}

Keywords: Suicide, attempted; Recidivism; Depression; Risk factors.

\section{Resumen}

Investigación cuyo objetivo es analizar la asociación entre la recurrencia del intento de suicidio y la gravedad de los síntomas depresivos. Se trata de una investigación exploratoria, descriptiva, cuantitativa, con recogida de datos realizada entre diciembre de 2017 y noviembre de 2019, en una Unidad de Urgencias de Assis / SP. Participaron 95 personas que asistieron por intento de suicidio, mediante entrevista con cuestionario semiestructurado y aplicación del Inventario de Depresión de Beck. Para el análisis de los datos se utilizó estadística descriptiva e inferencial, utilizando la prueba exacta de Fisher y el coeficiente de contingencia V de Cramer. El nivel de significancia se fijó en p <0,05. El proyecto de investigación fue aprobado por el Comité de Ética en Investigación. Entre los 95 (100,0\%) participantes, $66(69,5 \%)$ tuvieron una recurrencia del intento de suicidio y $36(37,9 \%)$ tenían un diagnóstico previo de depresión. Con el análisis de la asociación entre la recurrencia del intento de suicidio y los niveles de depresión, se permite afirmar que son variables dependientes tanto para personas sin depresión previa $(\mathrm{p}=0.022)$ como para 
personas con depresión previa $(\mathrm{p}=0.030)$. La recurrencia del intento de suicidio fue frecuente en personas con niveles más graves de síntomas depresivos.

Palabras clave: Intento de suicidio; Reincidencia; Depresión; Factores de riesgo.

\section{Introdução}

As estimativas da Organização Mundial de Saúde para as mortes anuais por suicídio no mundo são próximas a 800.000 (World Health Organization, 2019). No Brasil houve crescimento de 53,0\% na taxa de mortalidade por suicídio no período de 1990 a 2015, com registro total de 205.431 mortes por suicídio (Palma et al., 2020; Silva \& Marcolan, 2020).

O impacto sobre as famílias, amigos e comunidades é devastador e de longo alcance. Estudos apontam impacto emocional, social e econômico para cinco ou seis pessoas (World Health Organization, 2018; Franck et al., 2020), 60 pessoas (Penso \& Sena, 2020), e até 135 pessoas próximas (Cerel et al., 2019). Estima-se, ainda, que, ao longo da vida, cerca de $21 \%$ da população será exposta ao suicídio de pessoas próximas, sejam familiares, amigos ou conhecidos (Andriessen et al., 2017), e que esta exposição aumenta em 3,23 vezes as chances de suicídio e em 2,91 vezes as chances de ocorrer a tentativa de suicídio (Hill et al., 2020).

Há número bem maior para as tentativas de suicídio para cada morte. Estima-se que ocorram próximo a 20 tentativas de suicídio para cada morte de suicídio de adulto (World Health Organization, 2014). Em outra estimativa, a proporção entre morte por suicídio, tentativa de suicídio e ideação suicida é de 1:10:100 (McAuliffe, 2002).

Quando duas tentativas de suicídio, ou mais, são realizadas pela mesma pessoa, denomina-se reincidência, uma característica do comportamento suicida que assume fator de risco para a morte por suicídio, que pode ser ainda maior quando há intervalo de tempo menor entre as tentativas (Meira et al., 2020).

Por tentativa de suicídio compreende-se a ocorrência de ação autodestrutiva, com a intenção de morte, mas interrompida antes que a morte aconteça de fato, ou ação sem interrupção, porém sem a efetivação da morte como final da ação. A diferença entre outros comportamentos autodestrutivos e o suicídio se refere na intenção de colocar fim à própria vida (World Health Organization, 2000).

Os fatores de risco associados ao comportamento suicida (ideação suicida, tentativa de suicídio e suicídio) são inúmeros, fato que confere a condição multifatorial e multidimensional deste comportamento. Dentre esses fatores, a relação entre a depressão e o comportamento suicida é observada nas diversas pesquisas publicadas que abordam essa temática, por diferentes métodos de realização, sobretudo aos casos de transtorno depressivo grave, pois há maior probabilidade de o indivíduo apresentar ideias e atos suicidas (Moreira et al., 2020; Fernandes et al., 2020; Nascimento et al., 2020).

A depressão é um problema comum no mundo, com estimativa de mais de 300 milhões de pessoas afetadas em 2015, 4,4\% da população mundial, e previsão de ser a doença com maiores incidência e prevalência no mundo até o ano de 2030. No Brasil, 5,8\% da população é acometida pela depressão, o segundo maior índice de prevalência na região das Américas, atrás apenas dos Estados Unidos com 5,9\% (World Health Organization, 2017).

Neste contexto, a identificação e compreensão de prevalência do comportamento suicida relacionado a características dos indivíduos envolvidos permitem obter estratos de riscos nas populações para melhor acompanhamento, avaliação e prevenção do comportamento suicida (Félix et al., 2016).

No intuito de colaborar com essa perspectiva, esta pesquisa tem por objetivo analisar a associação entre a reincidência de tentativa de suicídio e a severidade da sintomatologia depressiva.

\section{Metodologia}

Trata-se de pesquisa exploratória, descritiva, com abordagem quantitativa, norteada pela ferramenta STROBE (von 
Elm et al., 2014) da rede Equator, com coleta de dados realizada no período de dezembro de 2017 a novembro de 2019, em uma Unidade de Pronto Atendimento na cidade de Assis, São Paulo.

As entrevistas foram realizadas com pessoas que apresentaram tentativa de suicídio e compreendeu os que estiveram em atendimento na Unidade de Pronto Atendimento do município de Assis/SP. Foram entrevistadas 113 pessoas que se conseguiu abordar e todos aceitaram participar da pesquisa dentre os que deram entrada para atendimento na Unidade de Pronto Atendimento, durante o período proposto para coleta dos dados assinalado acima.

Foram incluídos os que estavam em atendimento devido tentativa de suicídio, com condições cognitivas para participar da entrevista e maiores de 14 anos, sendo que os pacientes com idade a partir de 14 até 18 anos incompletos somente participaram com autorização também do responsável. Como critério de não inclusão, adotou-se a situação de o paciente ter recebido alta hospitalar antes do pesquisador chegar à unidade e abordá-lo com convite para participação da pesquisa. Foram excluídos 18 participantes e suas entrevistas foram desconsideradas em consequência da não resposta, por opção do participante, do Inventário de Depressão de Beck. Assim sendo, o número de entrevistados considerados foi de 95 (100,0\%).

Após a abordagem, a identificação do possível participante, explicações sobre a pesquisa, seus objetivos, formas de participação e consentimento na participação, foi entregue o Termo de Consentimento Livre e Esclarecido, assinado em duas vias pelo pesquisador e pelo participante, e posse de uma via para cada.

A coleta de dados se deu por meio de entrevista, com utilização de questionário semiestruturado elaborado pelos autores e do Inventário de Depressão de Beck (Gorestein \& Andrade, 1998).

O questionário semiestruturado continha informações sócio demográficas: sexo, idade, orientação sexual, cor de pele, estado civil, número de filhos, escolaridade, religião, condição de moradia, fonte de renda, participação em grupos sociais, diagnóstico de patologia física, diagnóstico de transtorno mental e uso de substâncias psicoativas, informações sobre os eventos que culminaram na tentativa de suicídio e no contexto de vida do participante.

No Inventário Depressão de Beck, para distinguir os níveis de depressão, adotamos escores para participantes que não tinham diagnóstico prévio escores entre 15 e 19 para disforia e escores acima de 20 para depressão. Nos participantes diagnosticados previamente, os escores adotados foram: sem depressão em resultado até 9 , depressão leve de 10 a 16 , depressão moderada de 17 a 29, e depressão grave ou severa de 30 a 63 (Williams, 1988).

Para processamento e análise dos dados as entrevistas foram digitadas em planilhas do Microsoft Excel e analisadas com estatística descritiva e inferencial. Para testar se as diferenças entre os grupos foram ou não significativas, utilizou-se o teste Exato de Fisher. Considerou-se as variáveis dependentes a reincidência da tentativa e suicídio e a classificação do nível de depressão obtida por meio do Inventário de Depressão de Beck, e como preditores as variáveis sócio demográficas. Para a análise da associação entre as variáveis reincidência de tentativa de suicídio e classificação dos níveis de depressão, empregouse o Coeficiente de Contingência V de Cramer. O nível de significância adotado para as análises deste estudo foi de $\mathrm{p}<0,05$, que transmite $95 \%$ de confiança para as afirmações.

As normas que regulamentam a ética em pesquisa com seres humanos, dispostas na Resolução no 466/2012 do Conselho Nacional de Saúde, foram asseguradas. O projeto de pesquisa foi submetido e aprovado pelo Comitê de Ética em Pesquisa da Universidade Federal de São Paulo, com Parecer n. ${ }^{\circ}$ 2.314.347, de 04 de outubro de 2017.

\section{Resultados}

No período de dezembro de 2017 a novembro de 2019 houve total de 309 ocorrências de tentativa de suicídio identificadas no município, destas, foram entrevistadas 113 pessoas que se conseguiu abordar, todas após atendimento de urgência na Unidade de Pronto Atendimento. Consideradas as perdas, a amostra é de 95 (100,0\%) participantes.

As características sócio demográficas dos participantes, classificados conforme a reincidência ou não da tentativa de 
suicídio, e presença ou não de diagnóstico prévio para depressão, estão dispostas na Tabela 1. É importante notar que há diferença estatística significante nas associações que se referem a depressão prévia entre as variáveis faixa etária ( $\mathrm{p}=0,001)$, estado civil $(\mathrm{p}<0001)$, filhos $(\mathrm{p}=0,017)$, religião $(\mathrm{p}=0,024)$ e fonte de renda $(\mathrm{p}=0,034)$.

Tabela 1. Frequências e percentuais para as características sócio demográficas e associação com a reincidência da tentativa de suicídio e depressão prévia ( $\mathrm{n}=95)$.

\begin{tabular}{|c|c|c|c|c|c|c|c|c|}
\hline \multirow[t]{2}{*}{ Característica } & \multirow[t]{2}{*}{ Nível } & \multirow[t]{2}{*}{ n $(\%)$} & \multicolumn{2}{|c|}{$\begin{array}{c}\text { Reincidência de } \\
\text { tentativa de suicídio }\end{array}$} & \multirow{2}{*}{$\begin{array}{c}\text { Valor } \\
\text { p* }^{*}\end{array}$} & \multicolumn{2}{|c|}{ Depressão prévia } & \multirow[t]{2}{*}{ Valor $\mathbf{p}^{* *}$} \\
\hline & & & Sim & Não & & Sem & Com & \\
\hline Total & - & $95(100,0)$ & $66(100,0)$ & $29(100,0)$ & - & $59(100,0)$ & $36(100,0)$ & - \\
\hline \multirow{2}{*}{ Sexo } & Feminino & $73(76,8)$ & $53(80,3)$ & $20(69,0)$ & \multirow{2}{*}{0,172} & $42(71,2)$ & $31(86,1)$ & \multirow{2}{*}{0,075} \\
\hline & Masculino & $22(23,2)$ & $13(19,7)$ & $9(31,0)$ & & $17(28,8)$ & $5(13,9)$ & \\
\hline \multirow{4}{*}{ Faixa etária } & 12 a 17 anos & $5(5,3)$ & $2(3,0)$ & $3(10,3)$ & \multirow{4}{*}{0,348} & $5(8,5)$ & $0(0,0)$ & \multirow{4}{*}{0,001} \\
\hline & 18 a 30 anos & $49(51,6)$ & $33(50,0)$ & $16(55,2)$ & & $37(62,7)$ & $12(33,3)$ & \\
\hline & 31 a 64 anos & $39(41,1)$ & $29(43,9)$ & $10(34,5)$ & & $17(28,8)$ & $22(61,1)$ & \\
\hline & 65 anos e + & $2(2,1)$ & $2(3,0)$ & $0(0,0)$ & & $0(0,0)$ & $2(5,6)$ & \\
\hline \multirow{3}{*}{ Orientação sexual } & Heterossexual & $85(89,5)$ & $59(89,4)$ & $26(89,7)$ & \multirow{3}{*}{0,309} & $53(89,8)$ & $32(88,9)$ & \multirow{3}{*}{0,563} \\
\hline & Bissexual & $9(9,5)$ & $7(10,6)$ & $2(6,9)$ & & $6(10,6)$ & $2(8,3)$ & \\
\hline & Homossexual & $1(1,1)$ & $0(0,0)$ & $1(3,4)$ & & $0(0,0)$ & $1(2,8)$ & \\
\hline \multirow{4}{*}{ Cor } & Branca & $48(50,5)$ & $31(47,0)$ & $17(58,6)$ & \multirow{4}{*}{0,186} & $29(49,2)$ & $19(52,8)$ & \multirow{4}{*}{0,894} \\
\hline & Parda & $41(43,2)$ & $32(48,5)$ & $9(31,0)$ & & $25(42,4)$ & $16(44,4)$ & \\
\hline & Preta & $5(5,3)$ & $3(4,5)$ & $2(6,9)$ & & $4(6,8)$ & $1(2,8)$ & \\
\hline & Amarela & $1(1,1)$ & $0(0,0)$ & $1(100,0)$ & & $1(100,0)$ & $0(0,0)$ & \\
\hline \multirow{4}{*}{ Estado civil } & Solteiro & $44(46,3)$ & $30(45,5)$ & $14(48,3)$ & \multirow{4}{*}{0,861} & $33(55,9)$ & $11(30,6)$ & \multirow{4}{*}{$<0,0001$} \\
\hline & Casado & $29(30,5)$ & $20(30,3)$ & $9(31,0)$ & & $17(28,8)$ & $12(33,3)$ & \\
\hline & Divorciado & $14(14,7)$ & $11(16,7)$ & $3(10,3)$ & & $2(3,4)$ & $12(33,3)$ & \\
\hline & União estável & $8(8,4)$ & $5(7,6)$ & $3(10,3)$ & & $7(11,9)$ & $1(2,8)$ & \\
\hline \multirow{2}{*}{ Filhos } & Sim & $60(63,2)$ & $44(66,7)$ & $16(55,2)$ & \multirow{2}{*}{0,200} & $32(54,2)$ & $28(77,8)$ & \multirow{2}{*}{$\mathbf{0 , 0 1 7}$} \\
\hline & Não & $35(36,8)$ & $22(33,3)$ & $13(44,8)$ & & $27(45,8)$ & $8(22,2)$ & \\
\hline \multirow{6}{*}{ Religião } & Católica & $35(36,8)$ & $24(36,4)$ & $11(37,9)$ & & $17(28,8)$ & $18(50,0)$ & \\
\hline & Evangélica & $35(36,8)$ & $25(37,9)$ & $10(34,5)$ & & $26(44,1)$ & $9(25,0)$ & \\
\hline & T. de Jeová & $2(2,1)$ & $1(1,5)$ & $1(3,4)$ & 0724 & $0(0,0)$ & $2(5,6)$ & \\
\hline & Espírita & $1(1,1)$ & $0(0,0)$ & $1(3,4)$ & 0,724 & $1(1,7)$ & $0(0,0)$ & 0,024 \\
\hline & Umbanda & $1(1,1)$ & $1(1,5)$ & $0(0,0)$ & & $0(0,0)$ & $1(2,8)$ & \\
\hline & Não & $21(22,1)$ & $15(22,7)$ & $6(20,7)$ & & $15(25,4)$ & $6(16,7)$ & \\
\hline & Não alfabetizado & $1(1,1)$ & $1(1,5)$ & $0(0,0)$ & & $1(1,7)$ & $0(0,0)$ & \\
\hline & EF incompleto & $21(22,1)$ & $14(21,2)$ & $7(24,1)$ & & $16(27,1)$ & $5(13,9)$ & \\
\hline & EF completo & $2(2,1)$ & $2(3,0)$ & $0(0,0)$ & & $0(0,0)$ & $2(5,6)$ & \\
\hline Escolaridade & EM incompleto & $17(17,9)$ & $10(15,2)$ & $7(24,1)$ & 0,502 & $11(18,6)$ & $6(16,7)$ & 0,349 \\
\hline & EM completo & $41(43,2)$ & $32(48,5)$ & $9(31,0)$ & & $23(39,0)$ & $18(50,0)$ & \\
\hline & ES incompleto & $7(7,4)$ & $4(6,1)$ & $3(10,3)$ & & $5(8,5)$ & $2(5,6)$ & \\
\hline & ES completo & $6(6,3)$ & $3(4,5)$ & $3(10,3)$ & & $3(5,1)$ & $3(8,3)$ & \\
\hline & Própria & $47(49,5)$ & $34(51,5)$ & $13(44,8)$ & & $34(57,6)$ & $13(36,1)$ & \\
\hline Fonte de renda & Dependente & $48(50,5)$ & $32(48,5)$ & $16(55,2)$ & 0,353 & $25(42,4)$ & $23(63,9)$ & $\mathbf{0 , 0 3 4}$ \\
\hline
\end{tabular}

* Teste Exato de Fisher para reincidência de tentativa de suicídio. ** Teste Exato de Fisher para depressão prévia

Fonte de dados: Elaborado pelos autores com dados da pesquisa (2021).

Na Tabela 2 apresentamos as análises de associação para os participantes sem depressão prévia. Para os grupos com reincidência, ou não, de tentativa de suicídio, houve significância com $\mathrm{p}<0,05$ para fonte de renda $(\mathrm{p}=0,026)$, relações familiares ( $\mathrm{p}=0,032)$, patologia física $(\mathrm{p}=0,044)$ e transtorno mental $(\mathrm{p}=0,004)$. Quanto a classificação do nível de depressão, a significância estatística quanto a cor de pele $(\mathrm{p}=0,001)$. 
Tabela 2. Frequências e percentuais para as características sócio demográficas - sem depressão prévia $(n=59)$.

\begin{tabular}{|c|c|c|c|c|c|c|c|c|}
\hline \multirow{2}{*}{ Característica } & \multirow[t]{2}{*}{ Nível } & \multicolumn{2}{|c|}{$\begin{array}{c}\text { Reincidência de } \\
\text { tentativa de suicídio }\end{array}$} & \multirow{2}{*}{$\begin{array}{c}\text { Valor } \\
\mathbf{p}^{*}\end{array}$} & \multicolumn{3}{|c|}{ Classificação IDB } & \multirow{2}{*}{$\begin{array}{c}\text { Valor } \\
\mathbf{p}^{* *}\end{array}$} \\
\hline & & Sim & Não & & Sem depressão & Disforia & Depressão & \\
\hline Total & - & $37(100,0)$ & $22(100,0)$ & - & $4(100,0)$ & $7(100,0)$ & $48(100,0)$ & - \\
\hline \multirow{2}{*}{ Sexo } & Feminino & $28(75,7)$ & $14(63,6)$ & \multirow[t]{2}{*}{0,244} & $4(100,0 \%)$ & $4(57,1 \%)$ & $34(70,8 \%)$ & \multirow{2}{*}{0,403} \\
\hline & Masculino & $9(24,3)$ & $8(36,4)$ & & $0(0,0 \%)$ & $3(42,9 \%)$ & $14(29,2 \%)$ & \\
\hline \multirow{3}{*}{ Faixa etária } & 12 a 17 anos & $2(5,4)$ & $3(13,6)$ & \multirow[t]{3}{*}{0,593} & $1(25,0 \%)$ & $1(14,3 \%)$ & $3(6,2 \%)$ & \multirow{3}{*}{0,328} \\
\hline & 18 a 30 anos & $24(64,9)$ & $13(59,1)$ & & $2(50,0 \%)$ & $3(42,9 \%)$ & $32(66,7 \%)$ & \\
\hline & 31 a 64 anos & $11(29,7)$ & $6(27,3)$ & & $1(25,0 \%)$ & $3(42,9 \%)$ & $13(27,1 \%)$ & \\
\hline \multirow{2}{*}{$\begin{array}{l}\text { Orientação } \\
\text { sexual }\end{array}$} & Heterossexual & $33(89,2)$ & $20(90,9)$ & \multirow[t]{2}{*}{0,603} & $4(100,0 \%)$ & $6(85,7 \%)$ & $43(89,6 \%)$ & \multirow{2}{*}{0,728} \\
\hline & Bissexual & $4(10,8)$ & $2(9,1)$ & & $0(0,0 \%)$ & $1(14,3 \%)$ & $5(10,4 \%)$ & \\
\hline \multirow{4}{*}{ Cor de pele } & Amarela & $0(0,0)$ & $1(4,5)$ & \multirow{4}{*}{0,138} & $0(0,0 \%)$ & $0(0,0 \%)$ & $1(2,1 \%)$ & \multirow{4}{*}{0,001} \\
\hline & Branca & $16(43,2)$ & $13(59,1)$ & & $3(75,0 \%)$ & $2(28,6 \%)$ & $24(50,0 \%)$ & \\
\hline & Parda & $19(51,4)$ & $6(27,3)$ & & $0(0,0 \%)$ & $2(28,6 \%)$ & $23(47,9 \%)$ & \\
\hline & Preta & $2(5,4)$ & $2(9,1)$ & & $1(25,0 \%)$ & $3(42,9 \%)$ & $0(0,0 \%)$ & \\
\hline \multirow{4}{*}{ Estado civil } & Casado & $10(27,0)$ & $7(31,8)$ & & $0(0,0)$ & $4(57,1)$ & $13(27,1)$ & \\
\hline & União estável & $5(13,5)$ & $2(9,1)$ & 0032 & $1(25,0)$ & $1(14,3)$ & $5(10,4)$ & \\
\hline & Divorciado & $1(2,7)$ & $1(4,5)$ & 0,932 & $0(0,0)$ & $0(0,0)$ & $2(4,2)$ & 0,355 \\
\hline & Solteiro & $21(56,8)$ & $12(54,5)$ & & $3(75,0)$ & $2(28,6)$ & $28(58,3)$ & \\
\hline Filhos & Sim & $21(56,8)$ & $11(50,0)$ & 0407 & $1(25,0)$ & $5(71,4)$ & $26(54,2)$ & \\
\hline & Não & $16(43,2)$ & $11(50,0)$ & 0,407 & $3(75,0)$ & $2(28,6)$ & $22(45,8)$ & 0,366 \\
\hline & Católica & $9(24,3)$ & $8(36,4)$ & & $2(50,0)$ & $1(14,3)$ & $14(29,2)$ & \\
\hline Religião & Evangélica & $17(45,9)$ & $9(40,9)$ & & $2(50,0)$ & $5(71,4)$ & $19(39,6)$ & \\
\hline & Espírita & $0(0,0)$ & $1(4,5)$ & 0,383 & $0(0,0)$ & $0(0,0)$ & $1(2,1)$ & 0,570 \\
\hline & Não & $11(29,7)$ & $4(18,2)$ & & $0(0,0)$ & $1(14,3)$ & $14(29,2)$ & \\
\hline & Não alfabetizado & $1(2,7)$ & $0(0,0)$ & & $0(0,0)$ & $0(0,0)$ & $1(2,1)$ & \\
\hline & EF incompleto & $10(27,0)$ & $6(27,3)$ & & $0(0,0)$ & $3(42,9)$ & $13(27,1)$ & \\
\hline Escolaridade & EM incompleto & $6(16,2)$ & $5(22,7)$ & & $1(25,0)$ & $3(42,9)$ & $7(14,6)$ & \\
\hline & EM completo & $16(43,2)$ & $7(31,8)$ & 0,817 & $2(50,0)$ & $1(14,3)$ & $20(41,7)$ & 0,320 \\
\hline & ES incompleto & $3(8,1)$ & $2(9,1)$ & & $0(0,0)$ & $0(0,0)$ & $5(10,4)$ & \\
\hline & ES completo & $1(2,7)$ & $2(9,1)$ & & $1(25,0)$ & $0(0,0)$ & $2(4,2)$ & \\
\hline & Própria, empregado & $15(40,5)$ & $3(13,6)$ & & $0(0,0)$ & $2(28,6)$ & $16(33,3)$ & \\
\hline Fonte de renda & Própria, autônomo & $11(29,7)$ & $5(22,7)$ & 0,026 & $1(25,0)$ & $3(42,9)$ & $12(25,0)$ & 0,516 \\
\hline & Pais, cônjuge e outros & $11(29,7)$ & $14(63,6)$ & & $3(75,0)$ & $2(28,6)$ & $20(41,7)$ & \\
\hline Relações & Negativa & $24(64,9)$ & $8(36,4)$ & 0032 & $1(25,0)$ & $2(28,6)$ & $29(60,4)$ & \\
\hline familiares & Positiva & $13(35,1)$ & $14(63,6)$ & $\mathbf{0 , 0 3}$ & $3(75,0)$ & $5(71,4)$ & $19(39,6)$ & 0,163 \\
\hline Mudança poder & Sim & $11(29,7)$ & $11(50,0)$ & & $1(25,0)$ & $2(28,6)$ & $19(39,6)$ & \\
\hline econômico & Não & $26(70,3)$ & $11(50,0)$ & 0,101 & $3(75,0)$ & $5(71,4)$ & $29(60,4)$ & 0,886 \\
\hline Participação em & Sim & $0(0,0)$ & $2(9,1)$ & & $1(25,0)$ & $0(0,0)$ & $1(2,1)$ & \\
\hline grupos sociais & Não & $37(100,0)$ & $20(90,9)$ & 0,135 & $3(75,0)$ & $7(100,0)$ & $47(97,9)$ & 0,144 \\
\hline Pat & Sim & $16(43,2)$ & $4(18,2)$ & 0044 & $1(25,0)$ & $0(0,0)$ & $19(39,6)$ & 103 \\
\hline & Não & $21(56,8)$ & $18(81,8)$ & & $3(75,0)$ & $7(100,0)$ & $29(60,4)$ & ,103 \\
\hline Transtorno & Sim & $21(56,8)$ & $4(18,2)$ & & $0(0,0)$ & $2(28,6)$ & $23(47,9)$ & \\
\hline mental & Não & $16(43,2)$ & $18(81,8)$ & & $4(100,0)$ & $5(71,4)$ & $25(52,1)$ & 0,135 \\
\hline & Sim & $15(40,5)$ & $5(22,7)$ & & $0(0,0)$ & $2(28,6)$ & $18(37,5)$ & 0439 \\
\hline com drogas & Não & $22(59,5)$ & $17(77,3)$ & 0,132 & $4(100,0)$ & $5(71,4)$ & $30(62,5)$ & 0,439 \\
\hline Situação & Sim & $36(97,3)$ & $21(95,5)$ & & $4(100,0)$ & $6(85,7)$ & $47(97,9)$ & \\
\hline traumática & Não & $1(2,7)$ & $1(4,5)$ & 1 & $0(0,0)$ & $1(14,3)$ & $1(2,1)$ & ,341 \\
\hline & Sim & $6(16,2)$ & $4(18,2)$ & & $0(0,0)$ & $2(28,6)$ & $8(16,7)$ & 064 \\
\hline Planejamento & Não & $31(83,8)$ & $18(81,8)$ & 0,557 & $4(100,0)$ & $5(71,4)$ & $40(83,3)$ & 0,645 \\
\hline Emissão de & Sim & $12(32,4)$ & $4(18,2)$ & & $1(25,0)$ & $3(42,9)$ & $12(25,0)$ & \\
\hline avisos & Não & $25(67,6)$ & $18(81,8)$ & 188 & $3(75,0)$ & $4(57,1)$ & $36(75,0)$ & 0,637 \\
\hline
\end{tabular}

* Teste Exato de Fisher para Reincidência de tentativa de suicídio. ** Teste Exato de Fisher para Classificação no IDB (Inventário de Depressão de Beck). Fonte de dados: Elaborado pelos autores com dados da pesquisa (2021).

Para os participantes com depressão prévia, apresentamos, na Tabela 3, as associações entre as variáveis. Para a reincidência de tentativa de suicídio, há diferença em relação a fonte de renda ( $\mathrm{p}=0,044)$. No nível de depressão, há diferença para a variável estado civil $(\mathrm{p}=0,013)$. 
Tabela 3. Frequências e percentuais para as características sócio demográficas - com depressão prévia (n=36).

\begin{tabular}{|c|c|c|c|c|c|c|c|c|}
\hline \multirow[t]{2}{*}{ Característica } & \multirow[t]{2}{*}{ Nível } & \multicolumn{2}{|c|}{$\begin{array}{c}\text { Reincidência de } \\
\text { tentativa de suicídio }\end{array}$} & \multirow[t]{2}{*}{ Valor $\mathbf{p}^{*}$} & \multicolumn{3}{|c|}{ Classificação IDB } & \multirow{2}{*}{$\begin{array}{c}\text { Valor } \\
\mathbf{p}^{* *}\end{array}$} \\
\hline & & Sim & Não & & Leve & Moderada & Grave & \\
\hline Total & - & $29(100,0)$ & $7(100,0)$ & - & $1(100,0)$ & $7(100,0)$ & $28(100,0)$ & - \\
\hline \multirow{2}{*}{ Sexo } & Feminino & $25(86,2)$ & $6(85,7)$ & \multirow{2}{*}{1,000} & $0(0,0 \%)$ & $7(100,0 \%)$ & $24(85,7 \%)$ & \multirow{2}{*}{0,116} \\
\hline & Masculino & $4(13,8)$ & $1(14,3)$ & & $1(100,0 \%)$ & $0(0,0 \%)$ & $4(14,3 \%)$ & \\
\hline \multirow{3}{*}{ Faixa etária } & 18 a 30 anos & $9(31,0)$ & $3(42,9)$ & \multirow{3}{*}{0,792} & $0(0,0 \%)$ & $3(42,9 \%)$ & $9(32,1 \%)$ & \multirow{3}{*}{0,878} \\
\hline & 31 a 64 anos & $18(62,1)$ & $4(57,1)$ & & $1(100,0 \%)$ & $4(57,1 \%)$ & $17(60,7 \%)$ & \\
\hline & 65 anos e + & $2(6,9)$ & $0(0,0)$ & & $0(0,0 \%)$ & $0(0,0 \%)$ & $2(7,1 \%)$ & \\
\hline \multirow{3}{*}{ Orientação sexual } & Heterossexual & $26(89,7)$ & $6(85,7)$ & \multirow{3}{*}{0,271} & $1(100,0 \%)$ & $7(100,0 \%)$ & $24(85,7 \%)$ & \multirow{3}{*}{1,000} \\
\hline & Homossexual & $0(0,0)$ & $1(14,3)$ & & $0(0,0 \%)$ & $0(0,0 \%)$ & $1(3,6 \%)$ & \\
\hline & Bissexual & $3(10,3)$ & $0(0,0)$ & & $0(0,0 \%)$ & $0(0,0 \%)$ & $3(10,7 \%)$ & \\
\hline \multirow{3}{*}{ Cor } & Branca & $15(51,7)$ & $4(57,1)$ & \multirow{3}{*}{1,000} & $0(0,0 \%)$ & $4(57,1 \%)$ & $15(53,6 \%)$ & \multirow{3}{*}{0,866} \\
\hline & Parda & $13(44,8)$ & $3(42,9)$ & & $1(100,0 \%)$ & $3(42,9 \%)$ & $12(42,9 \%)$ & \\
\hline & Preta & $1(3,4)$ & $0(0,0)$ & & $0(0,0 \%)$ & $0(0,0 \%)$ & $1(3,6 \%)$ & \\
\hline \multirow{4}{*}{ Estado civil } & Casado & $10(34,5)$ & $2(28,6)$ & \multirow{4}{*}{0,327} & $0(0,0 \%)$ & $5(71,4 \%)$ & $7(25,0 \%)$ & \\
\hline & União estável & $0(0,0)$ & $1(14,3)$ & & $1(100,0 \%)$ & $0(0,0 \%)$ & $0(0,0 \%)$ & \\
\hline & Divorciado & $10(34,5)$ & $10(34,5)$ & & $0(0,0 \%)$ & $1(14,3 \%)$ & $11(39,3 \%)$ & 0,013 \\
\hline & Solteiro & $9(31,0)$ & $2(28,6)$ & & $0(0,0 \%)$ & $1(14,3 \%)$ & $10(35,7 \%)$ & \\
\hline Filhos & Sim & $23(79,3)$ & $5(71,4)$ & 0.497 & $1(100,0 \%)$ & $7(100,0 \%)$ & $20(71,4 \%)$ & 0351 \\
\hline Filhos & Não & $6(20,7)$ & $2(28,6)$ & 0,491 & $0(0,0 \%)$ & $0(0,0 \%)$ & $8(28,6 \%)$ & 0,351 \\
\hline & Católica & $15(51,7)$ & $3(42,9)$ & & $1(100,0 \%)$ & $4(57,1 \%)$ & $13(46,4 \%)$ & \\
\hline & Evangélica & $8(27,6)$ & $1(14,3)$ & & $0(0,0 \%)$ & $0(0,0 \%)$ & $9(32,1 \%)$ & \\
\hline Religião & T. de Jeová & $1(3,4)$ & $1(14,3)$ & 0,564 & $0(0,0 \%)$ & $2(28,6 \%)$ & $0(0,0 \%)$ & 0,100 \\
\hline & Umbanda & $1(3,4)$ & $0(0,0)$ & & $0(0,0 \%)$ & $0(0,0 \%)$ & $1(3,6 \%)$ & \\
\hline & Não & $4(13,8)$ & $2(28,6)$ & & $0(0,0 \%)$ & $1(14,3 \%)$ & $5(17,9 \%)$ & \\
\hline & EF incompleto & $4(13,8)$ & $1(14,3)$ & & $0(0,0 \%)$ & $1(14,3 \%)$ & $4(14,3 \%)$ & \\
\hline & EF completo & $2(5,9)$ & $0(0,0)$ & & $0(0,0 \%)$ & $0(0,0 \%)$ & $2(7,1 \%)$ & \\
\hline & EM incompleto & $4(13,8)$ & $2(28,6)$ & & $1(100,0 \%)$ & $1(14,3 \%)$ & $4(14,3 \%)$ & \\
\hline Escolaridade & EM completo & $16(55,2)$ & $2(28,6)$ & 0,417 & $0(0,0 \%)$ & $4(57,1 \%)$ & $14(50,0 \%)$ & 0,80 \\
\hline & ES incompleto & $1(3,4)$ & $1(14,3)$ & & $0(0,0 \%)$ & $0(0,0 \%)$ & $2(7,1 \%)$ & \\
\hline & ES completo & $2(6,9)$ & $1(14,3)$ & & $0(0,0 \%)$ & $1(14,3 \%)$ & $2(7,1 \%)$ & \\
\hline & Própria & $8(27,6)$ & $5(71,4)$ & & $1(100,0)$ & $3(42,9)$ & $9(32,1)$ & \\
\hline Fonte de renda & Dependente & $21(72,4)$ & $2(28,6)$ & 0,044 & $0(0,0)$ & $4(57,1)$ & $19(67,9)$ & 0,487 \\
\hline Relações & Negativa & $24(82,8)$ & $5(71,4)$ & 0415 & $0(0,0 \%)$ & $5(71,4 \%)$ & $24(85,7 \%)$ & 0127 \\
\hline familiares & Positiva & $5(17,2)$ & $2(28,6)$ & 0,415 & $1(100,0 \%)$ & $2(28,6 \%)$ & $4(14,3 \%)$ & 0,127 \\
\hline Mudança poder & Sim & $18(62,1)$ & $5(71,4)$ & & $0(0,0 \%)$ & $5(71,4 \%)$ & $18(64,3 \%)$ & \\
\hline econômico & Não & $11(37,9)$ & $2(28,6)$ & 501 & $1(100,0 \%)$ & $2(28,6 \%)$ & $10(35,7 \%)$ & 0,606 \\
\hline Participação em & Sim & $4(13,8)$ & $0(0,0)$ & & $0(0,0 \%)$ & $0(0,0 \%)$ & $4(14,3 \%)$ & \\
\hline grupos sociais & Não & $25(86,2)$ & $7(100,0)$ & 403 & $1(100,0 \%)$ & $7(100,0 \%)$ & $24(85,7 \%)$ & 0,611 \\
\hline & Sim & $15(51,7)$ & $2(28,6)$ & & $0(0,0 \%)$ & $4(57,1 \%)$ & $13(46,4 \%)$ & \\
\hline & Não & $14(48,3)$ & $5(71,4)$ & 1 & $1(100,0 \%)$ & $3(42,9 \%)$ & $15(53,6 \%)$ & ,837 \\
\hline Envolvimento com & Sim & $12(41,4)$ & $4(57,1)$ & & $1(100,0 \%)$ & $5(71,4 \%)$ & $10(35,7 \%)$ & \\
\hline drogas & Não & $17(58,6)$ & $3(42,9)$ & 0,369 & $0(0,0 \%)$ & $2(28,6 \%)$ & $18(64,3 \%)$ & 0,103 \\
\hline Situação & Sim & $25(86,2)$ & $5(71,4)$ & & $0(0,0 \%)$ & $6(85,7 \%)$ & $24(85,7 \%)$ & \\
\hline traumática & Não & $4(13,8)$ & $2(28,6)$ & 0,329 & $1(100,0 \%)$ & $1(14,3 \%)$ & $4(14,3 \%)$ & ,233 \\
\hline & Sim & $8(27,6)$ & $2(28,6)$ & & $1(100,0 \%)$ & $0(0,0 \%)$ & $9(32,1 \%)$ & \\
\hline Planejamento & Não & $21(72,4)$ & $5(71,4)$ & 0,645 & $0(0,0 \%)$ & $7(100,0 \%)$ & $19(67,9 \%)$ & 0,051 \\
\hline & Sim & $11(37,9)$ & $1(14,3)$ & & $0(0,0 \%)$ & $0(0,0 \%)$ & $12(42,9 \%)$ & \\
\hline Emissão de avisos & Não & $18(62,1)$ & $6(85,7)$ & 0,235 & $1(100,0 \%)$ & $7(100,0 \%)$ & $16(57,1 \%)$ & 0,070 \\
\hline
\end{tabular}

* Teste Exato de Fisher para Reincidência de tentativa de suicídio. ** Teste Exato de Fisher para Classificação no IDB (Inventário de Depressão de Beck). Fonte de dados: Elaborado pelos autores com dados da pesquisa (2021).

Com a análise da associação entre a reincidência da tentativa de suicídio e os níveis de depressão, apresentada na Tabela 4, é permitido afirmar que são variáveis dependentes tanto para participantes sem depressão prévia (p=0,022), como para os com depressão prévia ( $\mathrm{p}=0,030)$, de forma que a reincidência para tentativa de suicídio é maior risco para participantes com níveis mais graves de sintomatologia depressiva. 
Tabela 4. Associação entre a reincidência da tentativa de suicídio e da classificação da sintomatologia depressiva $(\mathrm{n}=95)$.

\begin{tabular}{|c|c|c|c|c|c|c|}
\hline \multirow{2}{*}{$\begin{array}{l}\text { Depressão } \\
\text { prévia }\end{array}$} & \multirow[t]{2}{*}{ Classificação - IDB } & \multirow[t]{2}{*}{ n $(\%)$} & \multicolumn{2}{|c|}{$\begin{array}{l}\text { Reincidência de tentativa de } \\
\text { suicídio }\end{array}$} & \multirow[t]{2}{*}{$\rho$} & \multirow[t]{2}{*}{ Valor p* } \\
\hline & & & Sim - n (\%) & Não - n (\%) & & \\
\hline \multirow{4}{*}{$\begin{array}{l}\text { Sem depressão } \\
\text { prévia }\end{array}$} & Sem depressão & $4(6,8 \%)$ & $0(0,0)$ & $4(18,2)$ & \multirow{4}{*}{0,337} & \multirow{4}{*}{$\mathbf{0 , 0 2 2}$} \\
\hline & Disforia & $7(11,9 \%)$ & $4(10,8)$ & $3(13,9)$ & & \\
\hline & Depressão & $48(81,4 \%)$ & $33(89,2)$ & $15(68,2)$ & & \\
\hline & Total & $59(100,0)$ & $37(100,0)$ & $22(100,0)$ & & \\
\hline \multirow{4}{*}{$\begin{array}{l}\text { Com depressão } \\
\text { prévia }\end{array}$} & Depressão leve & $1(2,8 \%)$ & $0(0,0)$ & $1(14,3)$ & \multirow{4}{*}{0,425} & \multirow{4}{*}{$\mathbf{0 , 0 3 0}$} \\
\hline & Depressão moderada & $7(19,4 \%)$ & $4(13,8)$ & $3(42,9)$ & & \\
\hline & Depressão grave & $28(77,8 \%)$ & $25(86,2)$ & $3(42,9)$ & & \\
\hline & Total & $36(100,0)$ & $29(100,0)$ & $7(100,0)$ & & \\
\hline
\end{tabular}

* Coeficiente de contingência V de Cramer. Fonte de dados: Elaborado pelos autores com dados da pesquisa (2021).

\section{Discussão}

A tentativa de suicídio é entendida como um comportamento auto lesivo, potencialmente fatal, associado a intenção de morte. Entretanto, destaca-se que grande parte das vezes, a motivação de jovens para a tentativa de suicídio não é o morrer, mas interromper o sofrimento causado por uma situação intolerável, exteriorizar a hostilidade ou clamar por atenção (Turecki \& Brent, 2016).

Em situação de sofrimento e escassez, e até ausência, de ferramentas para a solução de problemas, a tentativa de suicídio é uma alternativa para a interrupção deste estado de aflição. Não ocorrendo a morte por suicídio, a ideação suicida pode perdurar por fatores como maiores níveis de ansiedade social, baixa percepção de auto eficácia, baixa autoestima e o relacionamento familiar prejudicado (Pereira et al., 2018), de forma que o risco para uma nova tentativa pode aumentar em cem vezes (Fontão et al., 2018).

A associação entre a tentativa de suicídio prévia e o risco elevado de morte posterior por suicídio é difundida na literatura (Hill et al., 2020; World Health Organization, 2014; Silva \& Marcolan, 2021), da mesma forma que se afirma que o suicídio pode ser prevenido quando há identificação dos fatores de risco e execução de plano de ações com esta finalidade (Turecki \& Brent, 2016; Miller \& Black, 2020).

Na investigação das mortes por suicídio, por meio da autópsia psicológica e psicossocial, alguns estudos evidenciam a existência de histórico de tentativa de suicídio, como no Piaú́ (Teixeira \& Martins, 2018), no Chile (Gómez et al., 2014), e na Colômbia (González et al., 2010), fato que evidencia o risco da repetição da ação auto lesiva.

Nesta pesquisa, a reincidência de tentativa de suicídio em participantes sem diagnóstico prévio para depressão foi maior em pessoas com renda própria, empregados assalariados $(\mathrm{p}=0,026)$, ambientes familiares com relações negativas $(\mathrm{p}=0,032)$, em tratamento para algum tipo de patologia física $(\mathrm{p}=0,044)$ e com transtornos mentais $(\mathrm{p}=0,004)$. Para participantes com diagnóstico prévio para depressão notou-se a maior ocorrência de reincidência de tentativa de suicídio em pessoas com dependência financeira por pais, cônjuges ou outros familiares ( $\mathrm{p}=0,044)$.

O comportamento suicida no trabalho pode ser entendido como expressão do sofrimento gerado no cenário laboral, uma grave consequência dos dramas sociais relacionados aos processos e formas organizacionais adotadas (Dejours \& Bègue, 2010).

São os processos e formas organizacionais que determinarão o sentido no trabalho. Trabalho que tem sentido é importante, é útil e é legítimo para aquele que o realiza. Dessa forma, o trabalho pode atribuir sentidos e significados positivos como justiça, possibilidade de segurança, autonomia, e relacionamentos satisfatórios, porém, também pode significar tortura e sofrimento, como uma obrigação e necessidade de sobrevivência. Assim, o trabalho deixa de significar realização pessoal e passa a ser entendido como um processo de desgaste físico-moral (Tolfo \& Piccinini, 2007). 
O sofrimento começa quando existe sentimento de desprazer e tensão, que ocorrem quando há o bloqueio da relação do trabalhador com a organização do trabalho. A organização negativa do trabalho envolve ações que incentivam o individualismo, o desmembramento entre os colegas e produtividade máxima como valor institucional, desvalorizando o trabalhador (Dejours, 2010).

É neste cenário de adoecimento que o suicídio está relacionado ao trabalho, como consequência de ações de humilhação, inibição, perseguição e menosprezo, atos ilegais do empregador que refletem negativamente na saúde física e psicológica (Oliveira \& Zempulski, 2018).

Sobre as relações familiares, sabe-se que no comportamento suicida há interferência das relações com familiares, com amigos e com outras pessoas com as quais haja relacionamento afetivo importante, de forma que o bom vínculo familiar assume papel de proteção ao comportamento suicida, enquanto que vínculos familiares conflituosos são potenciais fatores de risco (World Health Organization, 2014; Silva \& Marcolan, 2021). Com destaque para relações entre os membros da família e a estrutura familiar, verifica-se a associação em relação ao comportamento suicida (Veras, Silva, \& Katz, 2017).

Para a presença de patologia física, a dor, a doença crônica e o comportamento suicida apresentam uma interação mortal (World Health Organization, 2018). Doenças físicas como pulmonar, cardiovascular, endócrina e câncer, entre outras, estão associadas ao suicídio (Weber et al., 2017).

Uma revisão integrativa com o objetivo de verificar a relação entre a doença crônica não transmissível e o comportamento suicida no contexto hospitalar, apontou para a fragilidade emocional vivida por portadores de doenças crônicas não transmissíveis de tal modo que estes apresentam forte comportamento suicida (Magalhães \& Figueiredo, 2019).

Quanto aos transtornos mentais, tanto em países desenvolvidos quanto países em desenvolvimento percebe-se a existência de um transtorno mental, tratado ou não, e até mesmo não diagnosticado, na maioria dos casos de suicídio (World Health Organization, 2000; World Health Organization, 2018).

Dentre os inúmeros fatores de risco para o desenvolvimento do comportamento suicida, os transtornos mentais estão incluídos (Gili et al., 2018). Os de maior frequência e associação com o comportamento suicida são: depressão; transtorno de personalidade; alcoolismo; esquizofrenia; transtorno mental orgânico. Notabiliza-se que o risco do comportamento suicida é aumentado à medida que há sobreposição de comorbidades psiquiátricas (World Health Organization, 2000; World Health Organization, 2018; Magalhães \& Figueiredo, 2019).

Enfatiza-se que a depressão é o principal transtorno mental associado ao comportamento suicida (Roca et al., 2019), com associação do quadro clínico de depressão maior (World Health Organization, 2014; World Health Organization, 2017). Há risco 20 vezes maior para o suicídio em pessoas com transtorno depressivo maior (Eikelenboom et al., 2018).

Ao analisar a existência de diagnóstico prévio para depressão entre os participantes desta pesquisa, obteve-se que a depressão foi de maior incidência na população de meia idade $(\mathrm{p}=0,001)$. Esse fato pode se relacionar aos pensamentos a respeito da morte e aproximação do declínio físico (Costa et al., 2020), ou a adoção de comportamentos nocivos à saúde (Barros et al., 2017).

Para os participantes com diagnóstico prévio de depressão, divorciados e solteiros apresentaram maiores escores que indicam maior gravidade da sintomatologia depressiva $(\mathrm{p}=0,013)$.

Para o estado civil, há pesquisas que afirmam maior risco para depressão em pessoas sem companheiros (Silva \& Azeredo, 2019), solteiros, divorciados e viúvos (Organização Mundial da Saúde, 2000, 2018), contudo, os resultados desta pesquisa destacam essa ênfase na situação do divórcio ( $(\mathrm{p}<0,0001)$, o que sugere que a perda da companhia que já se teve é um fator de risco para o desenvolvimento da depressão.

A condição da falta de companhia de solteiros e divorciados se relaciona a solidão, que, por sua vez, é associada a depressão (Vink et al., 2008). Aos divorciados, à experiência traumática da perda conjugal agrega-se o processo do divórcio 
que assume característica danosa à saúde mental (Lage, 2018).

Obtivemos diferença estatisticamente significante em relação a não ter filhos e menor incidência de diagnóstico prévio para depressão $(\mathrm{p}=0,017)$, pois é um achado oposto ao apresentado na literatura, com afirmação sobre a tendência à depressão para pessoas sem filhos (Melo et al., 2020), e, por vezes, semelhante (Parreira et al., 2017).

Em relação a fonte de renda, os participantes que eram dependentes economicamente de familiares, apresentaram maior índice de depressão prévia $(\mathrm{p}=0,034)$. $\mathrm{O}$ desemprego pode ocasionar pobreza, diminuição do nível social, dificuldades domésticas e desesperança e, desta forma, é fortemente associado ao suicídio (Organização Mundial da Saúde, 2000). A essa situação de dificuldade financeira atrela-se a sensação de falta de controle do ambiente no qual se vive e a impotência para mudar a própria realidade. É marcada pela interferência, e até interrupção, da realização de projetos de vida (Santos et al., 2017). Para a questão financeira, a independência financeira possui relação com o senso de autonomia, de forma que a independência financeira permite a decisão sobre si e sobre seus bens. Na dependência financeira, o indivíduo se vê obrigado a seguir as ordenanças de outros, situação essa que tem potencial para desenvolver o comportamento suicida (Figueiredo et al., 2015).

$\mathrm{Na}$ análise sobre a gravidade da sintomatologia depressiva em participantes sem diagnóstico prévio de depressão, os participantes com cores de pele branca e parda foram os mais graves $(\mathrm{p}=0,001)$.

Estudo de revisão sistemática que se dedicou a identificar a tendência aos transtornos mentais conforme a cor de pele afirma que pessoas não brancas apresentam maior risco. Fato esse relacionado as interferências negativas que dificultam oportunidades educacionais, financeiras e sociais (Smolen \& Araújo, 2017). Há de se destacar que mulheres negras apresentam maior prevalência de transtornos mentais comuns (Smolen et al., 2018).

A associação entre o transtorno depressivo maior e o comportamento suicida não é uma novidade, pois a ideação suicida é um dos sintomas que definem esse transtorno, assim sendo, corrobora com a literatura existente (Eikelenboom et al., 2018).

É importante ressaltar que evidências recentes têm destacado a ocorrência de distúrbios cognitivos em pessoas deprimidas. Destas, 20 a $40 \%$ apresentam déficit nas funções executivas, de forma que retratam inabilidade cognitiva para controlar o comportamento em decorrência de pensamentos, das emoções e das ações - a rigidez cognitiva, que, por sua vez, resulta na falta de adaptação, na ruminação de pensamentos negativos e na dificuldade para positivar seus pensamentos. Tais fatos influenciam no desenvolvimento do comportamento suicida (Roca et al., 2019).

Estudo realizado no Canadá com pessoas com histórico de comportamento suicida (ideação ou tentativa) evidenciou que a interrupção do episódio de depressão maior esteve associada a maior chance para remissão do comportamento suicida (Fuller-Thomson et al., 2019).

Um estudo holandês que por seis anos acompanhou pessoas diagnosticadas com transtorno depressivo maior afirma a importância do acompanhamento e tratamento de pessoas com depressão, contudo, na perspectiva multifatorial do comportamento suicida, não pode ser a única ação para prevenir a tentativa de suicídio e a morte por suicídio. O risco de tentativa de suicídio futura em pessoas com depressão esteve relacionado a história de tentativa de suicídio, ideações suicidas, insônia, uso de antidepressivos e desvantagens socioeconômicas (Eikelenboom et al., 2018).

Destaca-se ser a depressão considerada um fator de risco para o desenvolvimento do comportamento suicida, e que, como os demais fatores de risco, não deve ser entendida de forma isolada como única influenciadora para o desenvolvimento deste comportamento, mas compreendida como integrante da característica multifatorial do comportamento suicida. Em outras palavras, depressão e suicídio são distintos, mas que coexistem influenciando-se mutuamente (Assumpção et al., 2018).

Ainda neste contexto, assegura-se que não é correto afirmar que todas as pessoas com depressão maior apresentarão comportamento suicida, e, da mesma forma, não se pode afirmar que todo comportamento suicida abriga a ocorrência 
depressiva (Assumpção et al., 2018), no entanto, os dados apresentados evidenciam que há associação entre esses fenômenos, com ênfase aos níveis graves/severos da sintomatologia depressiva.

\section{Considerações Finais}

A reincidência de tentativa de suicídio nas unidades de atendimento às urgências é uma realidade, e evidencia o risco de morte por suicídio quando não há tratamento adequado aos pacientes, pelos altos índices de repetição desta ação, que nesta pesquisa foi de $69,5 \%$.

A depressão, um dos transtornos mentais de maior incidência e prevalência no mundo, é subdiagnosticada e consequentemente não tratada. Aos participantes desta pesquisa, 36 (37,9\%) afirmaram o diagnóstico prévio para depressão, contudo, ao aplicar o Inventário de Depressão de Beck, 84 (88,4\%) participantes apresentaram resultado indicativo de depressão, com ênfase aos níveis mais graves.

Ao analisar a associação entre a reincidência da tentativa de suicídio e os níveis de depressão, tanto para pessoas sem depressão prévia $(\mathrm{p}=0,022)$, como para pessoas com depressão prévia $(\mathrm{p}=0,030)$, podemos afirmar que a reincidência para tentativa de suicídio foi mais prevalente para pessoas com níveis mais graves de sintomatologia depressiva.

A partir desses resultados, sugere-se futuros estudos que se dediquem à traçar estratégias eficazes para o diagnóstico da depressão, e a compreensão da reincidência da tentativa de suicídio.

\section{Agradecimentos}

O presente trabalho foi realizado com apoio da Coordenação de Aperfeiçoamento de Pessoal de Nível Superior Brasil (CAPES) - Código de Financiamento 001.

\section{Referências}

Andriessen, K., Rahman, B., Draper, B., Dudley, M., \& Mitchell, P. B. (2017). Prevalence of exposure to suicide: a meta-analysis of population-based studies. Journal of Psychiatric Research, 88, 113-120. https://doi.org/10.1016/j.jpsychires.2017.01.017

Assumpção, G. L. S., Oliveira, L. A., \& Souza, M. F. S. (2018). Depressão e suicídio: uma correlação. Pretextos, 3(5), $312-333$.

Barros, M. B. A., Lima, M. G., Azevedo, R. C. S., Medina, L. B. P., Lopes, C. S., \& Malta, D. C. (2017). Depressão e comportamentos de saúde em adultos brasileiros - PNS 2013. Revista de Saúde Pública, 51(Supl 1), 8s. https://doi.org/10.1590/S1518-8787.2017051000084

Cerel, J., Brown, M. M., Maple, M., Singleton, M., van de Venne, J., Moore, M., \& Flaherty, C. (2019). How many people are exposed to suicide? Not six. Suicide and Life-Threatening Behavior, 49(2), 529-534. https://doi.org/10.1111/sltb.12450

Costa, S. M., Ramos, F. C. N., Barbosa, E., \& Santos, N. B. d. (2020). Aspectos sociais das relações entre depressão e isolamento dos idosos. GIGAPP estudios, 7, 150-165.

Dejours, C., \& Bègue, F. (2010). Suicídio e trabalho: o que fazer? Sobradinho (DF): Paralelo 15.

Eikelenboom, M., Beekman, A., Penninx, B., \& Smit, J. H. (2019). A 6-year longitudinal study of predictors for suicide attempts in major depressive disorder. Psychological medicine, 49(6), 911-921. https://doi.org/10.1017/S0033291718001423

Félix, T. A., Oliveira, E. N., Lopes, M. V. de O., Parente, J. R. F., Dias, M. S. de A., \& Moreira, R. M. M. (2016). Fatores de risco para tentativa de suicídio: produção de conhecimento no Brasil. Revista Contexto \&Amp; Saúde, 16(31), 173-185. https://doi.org/10.21527/2176-7114.2016.31.173-185

Fernandes, M. A., Silva, J. S., Campos, L. R. B., Nepomuceno, V. M. S., Vasconcelos, A. C. B., \& Oliveira, A. L. C. B. (2020). Prevenção ao suicídio: vivências de estudantes universitários. Revista Cuidarte, 11(2), e791. https://doi.org/10.15649/cuidarte.791

Figueiredo, A. E. B., Silva, R. M., Vieira, L. J. E. S., Mangas, R. M. N., Sousa, G. S., Freitas, J. S., \& Sougey, E. B. (2015). É possível superar ideações e tentativas de suicídio? Um estudo sobre idosos. Ciência \& Saúde Coletiva, 20(6), 1711-1719. https://doi.org/10.1590/1413-81232015206.02102015

Fontão, M. C., Rodrigues, J., Lino, M. M., \& Kempfer, S. S. (2018). Nursing care to people admitted in emergency for attempted suicide. Revista Brasileira de Enfermagem, 71(Suppl 5), 2199-2205. https://doi.org/10.1590/0034-7167-2017-0219

Franck, M. C., Monteiro, M. G., \& Limberger, R. P. (2020). Mortalidade por suicídio no Rio Grande do Sul: uma análise transversal dos casos de 2017 e 2018. Epidemiologia e Serviços de Saúde, 29(2), e2019512. https://doi.org/10.5123/S1679-49742020000200014 
Fuller-Thomson, E., West, K. J., \& Baiden, P. (2019). The tide does turn: predictors of remission from suicidal ideation and attempt among Canadians who previously attempted suicide. Psychiatry Research, 274, 313-321. https://doi.org/10.1016/j.psychres.2019.02.030

Gili, M., Castellví, P., Vives, M., de la Torre-Luque, A., Almenara, J., Blasco, M. J., ... \& Roca, M. (2019). Mental disorders as risk factors for suicidal behavior in young people: A meta-analysis and systematic review of longitudinal studies. Journal of affective disorders, 245 , $152-162$. https://doi.org/10.1016/j.jad.2018.10.115

Gómez, Ch. A., Opazo P, R., Levi A, R., Gómez Ch, M. S., Ibáñez H, C., \& Núñez M, C. (2014). Autopsias psicológicas de treinta suicidios en la IV Región de Chile. Revista chilena de neuro-psiquiatría, 52(1), 9-19. https://dx.doi.org/10.4067/S0717-92272014000100002

González, A., Rodríguez Betancur, Á., Aristizábal, A., García Valencia, J., Palacio, C., \& López Jaramillo, C. (2010). Suicidio y género en Antioquia (Colombia): estudio de autopsia psicológica. Revista Colombiana de Psiquiatría, 39(2), 251-267.

Gorestein, C., \& Andrade, L. (1998). Inventário de depressão de Beck: propriedades psicométricas da versão em português. Revista Psiquiatria Clínica São Paulo, 25(5), 245-250.

Hill, N. T. M., Robinson, J., Pirkis, J., Andriessen, K., Krysinska, K., Payne, A., \& Lampit, A. (2020). Association of suicidal behavior with exposure to suicide and suicide attempt: A systematic review and multilevel meta-analysis. PLoS Medicine, 17(3), e1003074. https://doi.org/10.1371/journal.pmed.1003074

Lage, M. J. O. S. (2018). O processo de divórcio e a coparentalidade - Um estudo qualitativo com pais divorciados. 2018.41 f. Dissertação (Mestrado em Psicologia Clínica e da Saúde) - Universidade Lusófona do Porto, Porto.

Magalhães, K. S., \& Figueiredo, A. E. B. (2019). Doenças crônicas não transmissíveis e sua relação com o comportamento suicida no âmbito hospitalar. Ciência, Cuidado e Saúde, 18(3), e45788. https://doi.org/10.4025/cienccuidsaude.v18i3.45788

McAuliffe, C. M. (2002). Suicidal Ideation as an Articulation of Intent: A Focus for Suicide Prevention? Archives of Suicide Research, 6(4), 325-338. https://doi.org/10.1080/13811110214524

Meira, S. S., Vilela, A. B. A., Lopes, C. R. S., Pereira, H. B. B., \& Alves, J. P. (2020). Representações sociais de profissionais de emergência sobre prevenção de readmissões hospitalares por tentativa de suicídio. Trabalho, Educação e Saúde, 18(3), e00276108. https://doi.org/10.1590/1981-7746-sol00276

Melo, M. G., Santos, R. P., Tavares, S. S., Saraiva, A. P. C., Carvalho, A. K. N., \& Silva, F. C. C. (2020). Aplicação da escala de depressão geriátrica abreviada em idosos ativos e sedentários do HIPERDIA. Revista Eletrônica Acervo Saúde, 12(9), e4214. https://doi.org/10.25248/reas.e4214.2020

Miller, J. N., \& Black, D. W. (2020). Bipolar Disorder and Suicide: a Review. Current psychiatry reports, 22(2), 6. https://doi.org/10.1007/s11920-020-1130-0

Moreira, R. M. M., Oliveira, E. N., Lopes, R. E., Lopes, M. V. de O., Félix, T. A., \& Oliveira, L. da S. (2020). Transtorno mental e risco de suicídio em usuários de substâncias psicoativas: uma revisão integrativa. SMAD Revista Eletrônica Saúde Mental Álcool E Drogas, 16(1), 1-10. https://doi.org/10.11606//issn.1806-6976.smad.2020.158433

Nascimento, V. S., Santos, A. V., Arruda, S. B., Silva, G. A., Cintra, J. D. S., Pinto, T. C. C., \& Ximenes, R. C. C. (2019). Association between eating disorders, suicide and depressive symptoms in undergraduate students of health-related courses. einstein (São Paulo), 18, eAO4908. https://doi.org/10.31744/einstein_journal/2020AO4908

Oliveira, A. M. B., \& Zempulski, T. L. (2018). O suicídio cometido em razão do meio ambiente de trabalho. Caderno da Escola Superior de Gestão Pública, Política, Jurídica e Segurança, 1(2), 181-208.

Organização Mundial da Saúde. (2000). Prevenção do suicídio: um manual para médicos clínicos gerais. OMS.

Palma, D. C. A., Santos, E. S., \& Ignotti, E. (2020). Análise dos padrões espaciais e caracterização dos suicídios no Brasil entre 1990 e 2015. Cadernos de Saúde Pública, 36(4), e00092819. https://doi.org/10.1590/0102-311X00092819

Parreira, B. D. M., Goulart, B. F., Ruiz, M. T., Silva, S. R., \& Gomes-Sponholz, F. A. (2017). Sintomas de depressão em mulheres rurais: fatores sociodemográficos, econômicos, comportamentais e reprodutivos. Acta Paulista de Enfermagem, 30(4), 375-382. https://doi.org/10.1590/19820194201700056

Penso, M. A., \& Sena, D. P. A. (2020). A desesperança do jovem e o suicídio como solução. Sociedade e Estado, 35(1), 61-81. https://doi.org/10.1590/s01026992-202035010004

Pereira, A. S., Willhelm, A. R., Koller, S. H., \& Almeida, R. (2018). Risk and protective factors for suicide attempt in emerging adulthood. Fatores de risco e proteção para tentativa de suicídio na adultez emergente. Ciencia \& saude coletiva, 23(11), 3767-3777. https://doi.org/10.1590/1413812320182311.29112016

Roca, M., Del Amo, A. R., Riera-Serra, P., Pérez-Ara, M. A., Castro, A., Roman Juan, J., García-Toro, M., García-Pazo, P., \& Gili, M. (2019). Suicidal risk and executive functions in major depressive disorder: a study protocol. BMC psychiatry, 19(1), 253. https://doi.org/10.1186/s12888-019-2233-1

Santos, M. S. P., Silva, T. P. S., Pires, C. M. C., Ramos, P. G. X., \& Sougey, E. B. (2017). Identificação de aspectos associados à tentativa de suicídio por envenenamento. Jornal Brasileiro de Psiquiatria, 66(4), 197-202. https://doi.org/10.1590/0047-2085000000171

Silva, A. N., \& Azeredo, C. M. (2019). The association between victimization resulting from intimate partner violence and depression among Brazilian adults. Ciencia \& saude coletiva, 24(7), 2691-2700. https://doi.org/10.1590/1413-81232018247.25002017

Silva, D. A., \& Marcolan, J. F. (2021). Suicide Attempts and Suicide in Brazil: An Epidemiological Analysis. Florence Nightingale J Nurs, 29, 294-302. https://doi.org/10.5152/FNJN.2021.21035 
Research, Society and Development, v. 10, n. 16, e299101623843, 2021

(CC BY 4.0) | ISSN 2525-3409 | DOI: http://dx.doi.org/10.33448/rsd-v10i16.23843

Silva, D. A., \& Marcolan, J. F. (2021). O impacto das relações familiares no comportamento suicida. Research, Society and Development, 10(2), e17310212349. https://doi.org/10.33448/rsd-v10i2.12349

Silva, D. A. da, \& Marcolan, J. F. (2020). Epidemiologia do suicídio no Brasil entre 1996 e 2016 e a política pública. Research, Society and Development, 9(2), e79922080. https://doi.org/10.33448/rsd-v9i2.2080

Smolen, J. R., \& Araújo, E. M. (2017). Race/skin color and mental health disorders in Brazil: a systematic review of the literature. Ciencia \& saude coletiva, 22(12), 4021-4030. https://doi.org/10.1590/1413-812320172212.19782016

Smolen, J. R., de Araújo, E. M., de Oliveira, N. F., \& de Araújo, T. M. (2018). Intersectionality of Race, Gender, and Common Mental Disorders in Northeastern Brazil. Ethnicity \& disease, 28(3), 207-214. https://doi.org/10.18865/ed.28.3.207

Teixeira, S. M. O., \& Martins, J. C. (2018). O. O suicídio de idosos em Teresina: fragmentos de autópsias psicossociais. Fractal: Revista de Psicologia, 30(2), $262-270$.

Tolfo, S. R., \& Piccinini, V. (2007). Sentidos e significados do trabalho: explorando conceitos, variáveis e estudos empíricos brasileiros. Psicologia \& Sociedade, 19(n. spe), 38-46. https://doi.org/10.1590/S0102-71822007000400007

Turecki, G., \& Brent, D. A. (2016). Suicide and suicidal behaviour. Lancet (London, England), 387(10024), 1227-1239. https://doi.org/10.1016/S01406736(15)00234-2

Veras, J. L. A., Silva, T. P. S., \& Katz, C. T. (2017). Funcionamento familiar e tentativa de suicídio entre adolescentes. Cadernos Brasileiros de Saúde Mental, 9(22), 70-82.

Vink, D., Aartsen, M. J., \& Schoevers, R. A. (2008). Risk factors for anxiety and depression in the elderly: a review. Journal of affective disorders, 106(1-2), 29-44. https://doi.org/10.1016/j.jad.2007.06.005

von Elm, E., Altman, D. G., Egger, M., Pocock, S. J., Gøtzsche, P. C., Vandenbroucke, J. P., \& STROBE Initiative (2014). The Strengthening the Reporting of Observational Studies in Epidemiology (STROBE) Statement: guidelines for reporting observational studies. International journal of surgery (London, England), 12(12), 1495-1499. https://doi.org/10.1016/j.ijsu.2014.07.013

Weber, A. N., Michail, M., Thompson, A., \& Fiedorowicz, J. G. (2017). Psychiatric Emergencies: Assessing and Managing Suicidal Ideation. The Medical clinics of North America, 101(3), 553-571. https://doi.org/10.1016/j.mcna.2016.12.006

Williams J. B. (1988). A structured interview guide for the Hamilton Depression Rating Scale. Archives of general psychiatry, 45(8), 742-747. https://doi.org/10.1001/archpsyc.1988.01800320058007

World Health Organization. (2019). Suicide in the world: Global Health Estimates. WHO.

World Health Organization. (2018). Suicide prevention.: WHO.

World Health Organization. (2017). Depression and other common mental disorders: global health estimates. WHO.

World Health Organization. (2014). Preventing suicide: A global imperative. WHO.

World Health Organization. (2000). Preventing suicide: a resource series. WHO. 